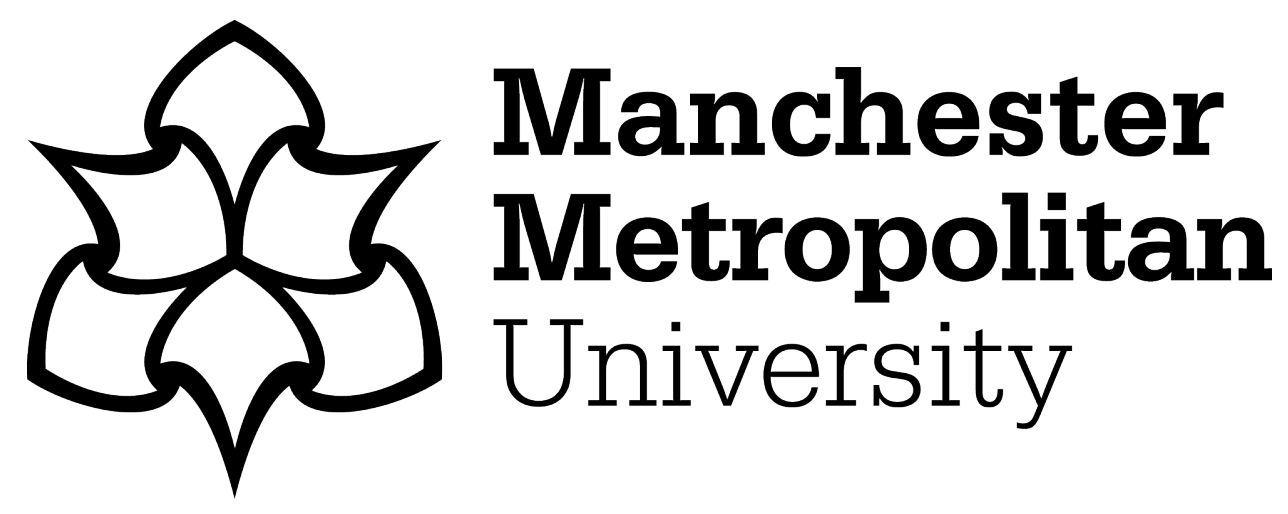

Weerasooriya, L, Mei, TX, Li, H ORCID logoORCID: https://orcid.org/00000002-8479-0091 and Luo, Y (2020) Application of State Estimators in Active Control of Railway Wheelsets. In: Advances in Dynamics of Vehicles on Roads and Tracks Proceedings of the 26th Symposium of the International Association of Vehicle System Dynamics, IAVSD 2019, August 12-16, 2019, Gothenburg, Sweden.

Downloaded from: https://e-space.mmu.ac.uk/626980/

Publisher: Springer

DOI: https://doi.org/10.1007/978-3-030-38077-9_6

Please cite the published version 


\title{
Application of State Estimators in Active Control of Railway Wheelsets
}

\author{
Lushan Weerasooriya ${ }^{1}$, T. X Mei ${ }^{1}$, Hong $\mathrm{Li}^{2}$ and Y Luo ${ }^{3}$ \\ ${ }^{1}$ University of Salford, Salford M5 4WT, United Kingdom \\ ${ }^{2}$ Manchester Metropolitan University, Manchester M15 6BH, United Kingdom \\ ${ }^{3}$ Tongji University, Shanghai, P R China \\ L.Weerasooriya@edu.salford.ac.uk
}

\begin{abstract}
This paper presents the development of a state observer for the estimation of output torque of an electromechanical actuator in the application of active wheelset control for railway vehicles. The output from the state estimator is essential to ensure that actuator responds appropriately and deliver accurate and fast control effort as demanded to maintain the stability of wheelsets. The formulation and design of the observer is based on the use of the actuator model only, so that it reduces substantially the complexity and difficult uncertainties related to the model of a full rail vehicle. The performance and robustness assessments of the state estimator integrated active control system are carried out with the use of a full bogie vehicle model.
\end{abstract}

Keywords: Active Wheelset Control, Actuator Dynamics, State Estimators, Torque Sensing

\section{Introduction}

Active control for railway wheelset can help to substantially reduce wear and tear of wheels and track surfaces by lowering creep forces [1]. In the design of such active control systems, in addition to the main wheelset controller, local controllers for the actuators are also used to ensure a fast and accurate delivery of the control effort demanded by the wheelset control system. However, the feedback of the output torque of the actuators applied at the wheelset can be very difficult to measure reliably in practice due to the harsh working conditions. This study presents the development of a model based approach to provide the estimation of the output torque. The proposed solution does not involve the vehicle dynamics which can be very complex - the design is based on the local actuator dynamics only and the simple measurements of the actuator current and velocity are used.

There are many previous studies conducted on the use of model based estimation techniques on railway vehicles with actively controlled suspension systems in order to estimate measurements which are difficult/impractical to obtain with readily available sensors. A research has focused on the use of a kalman filter to estimate the cant angle 
of a track using measurements from accelerometers and gyros mounted on the railway vehicle and wheelset accordingly [2] in both random and deterministic tracks. Another similar study has shown the application of using estimation techniques to obtain lateral displacement and yaw angle of wheelsets along with track condition such as curvature and cant angle [3]. In addition, a research has been done on use of multiple kalman filters to estimate creep coefficients and a kalman-bucy filter for the estimation of creep forces and interpretation of estimates through post-processing has enabled the detection of lateral and yaw creep forces [4]. Similarly, estimation techniques have been used in a semi-active control system to estimate torque and damping values of a vehicle suspensions [5] where an unscented kalman filter is used in the study to estimate vehicle suspension states such as vertical velocity of stiffness components while its vertical acceleration and stroke velocity are provided as measurable states which assist in developing a sliding mode controller.

In this study, a full active wheelset control scheme involving a wheelset controller and an actuator controller for each wheelset is developed for a full bogie vehicle model with the addition of having a state observer to provide the torque feedback to the actuator controller. The observer is developed based on the simple actuator model only, and it does not involve the dynamics of the vehicle which would result in a much more complex solution. The observer is formulated in such a way to consist of two states (the motor speed and toque applied to the wheelset). The measurements of the motor current and speed measurement used for correcting estimation errors in the observer are much easier to obtain accurately and reliably compared to the measurement of the torque output at the wheelset.

Performance of the state observer is assessed using a full bogie vehicle model with a number of different track inputs including the curves and straight track with irregularities. The robustness of the observer is also evaluated to account for the uncertainties and inaccuracies of the actuator parameters used in the observer.

\section{$2 \quad$ Active Control}

As illustrated in Figure 1, a conventional railway bogie vehicle model with solid-axle wheelsets and with an active wheelset control scheme is used in the study. Only the yaw and lateral dynamic motions [6] of the wheelsets and those of the bogie/body frames are taken into account in this model since the other dynamics such as vertical, pitch and roll movements are largely dynamically decoupled from the plan view motions and therefore not expected to have any substantial effect in this case.

In order to assess the performance and evaluate the performances of the observer estimations and its impact on actuator dynamics/controller, a number of different track conditions are used in the study, including a curved track with a curve radius of $300 \mathrm{~m}$ and a cant angle of 6 degrees at a relatively low speed of $25 \mathrm{~m} / \mathrm{s}$ and a straight track with generically generated irregularities at the high speed $83 \mathrm{~m} / \mathrm{s}$. 


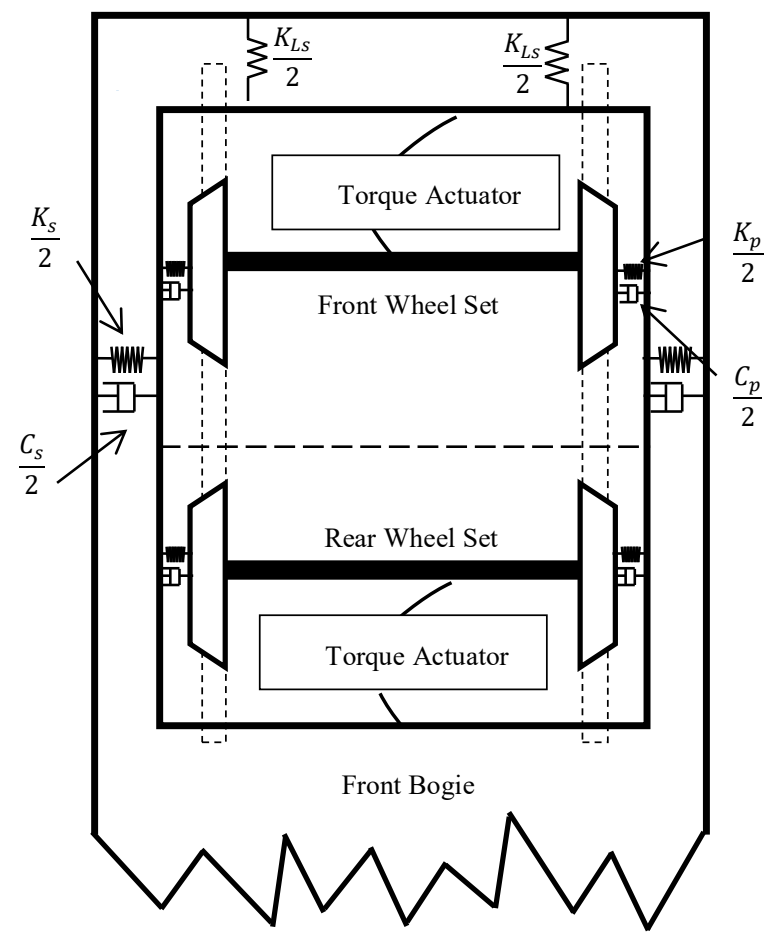

Front Half of the Train Body

Fig. 1. Active Primary Suspension - Full Bogie Model

The actuation for each wheelset is provided by an electro-mechanical actuator. As depicted in Figure 2 below, a DC electrical motor and a gearbox is used as the actuator model in the investigation. Parameters of the actuator such as gear ratio (n), stiffness $\left(\mathrm{K}_{\mathrm{g}}\right)$ and the damping $\left(\mathrm{C}_{\mathrm{g}}\right)$ at the gear wheel-load connection is selected after a thorough comparison of the performance to obtain optimum results. Equations 1-4 represent the dynamics of the electrical motor and the mechanical coupling with the wheelset.

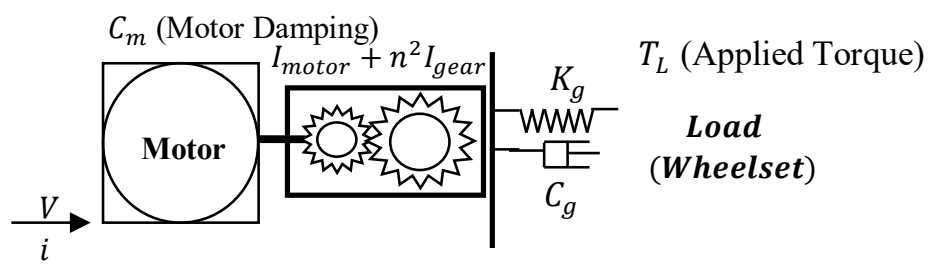

Fig. 2. The electro-mechanical actuator 


$$
\begin{aligned}
& \dot{i_{a}}=-\frac{r_{\text {arm }}}{l_{\text {arm }}} i_{a}-\frac{k_{e}}{l_{\text {arm }}} \dot{\theta_{m}}+\frac{1}{l_{\text {arm }}} v_{a} \\
& \left(I_{m}+I_{g} n^{2}\right) \ddot{\theta_{m}}=k_{t} i_{a}-c_{m} \dot{\theta_{m}}-n T_{L} \\
& \left(I_{m}+I_{g} n^{2}\right) \ddot{\theta_{m}}=k_{t} \dot{i}_{a}-c_{m} \dot{\theta_{m}}-C_{g} n^{2} \dot{\theta_{m}}-K_{g} n^{2} \theta_{m}+n K_{g} \psi_{m}+n C_{g} \dot{\psi_{m}} \\
& \text { Torque }\left(T_{L}\right)=K_{g} n \theta_{m}+C_{g} n \dot{\theta_{m}}-K_{g} \psi_{m}-C_{g} \dot{\psi_{m}}
\end{aligned}
$$

A number of different control strategies such as classical feedback controllers or model based optimal controllers have been previously proposed to provide stability control without interfering the natural curving of the solid axle wheelset [6-9], although there are also control schemes that are proposed to provide steering actions on curves to overcome the constraint on curving caused by passive suspensions [10]. In this study, the concept of absolute yaw stiffness method is applied to generate the torque required to stabilise the wheelsets as shown in Figure 3 while allowing the natural curving movements of the wheelsets [9].

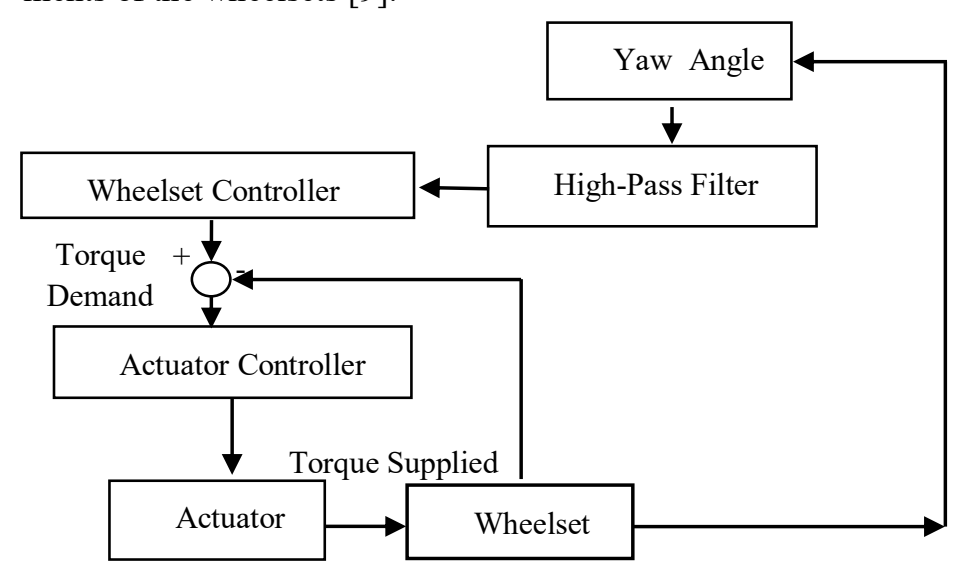

Fig. 3. Wheelset Controller and Actuator Controller

Two feedback control loops for each wheelset are used - the wheelset controller generates a torque demand for stabilising the wheelset whereas the local actuator controller ensures the motor to apply the required toque to the wheelset. Because the wheelset stability control is of high bandwidth in nature, the actuator controller is considered essential for the delivering of control efforts with fast and accurate responses. 


\section{State Observers}

The actuator controller requires the feedback of the actuator output torque applied at the wheelset which is very difficult to measure reliably due to the harsh operating environments of wheelsets. This study develops a model-based approach to estimate the required feedback from sensors that are much more reliable and easier to install. The proposed approach for the design is based on the dynamic relationship between the actuator and the torque applied to the wheelsets using the readily available measurements such motor current, and motor speed. This is a much simplified approach compared with the full state estimation that would also need to include the vehicle dynamics that can be potential very complex.

The simplified observer is formulated to have only two states - the rotor speed of the electric motor $\left(\dot{\theta}_{m}\right)$ and the torque applied at the wheelset $\left(T_{L}\right)$. The output measurement is the rotor speed and there is an additional input of the motor current that is also easily obtainable. The formulation and design process are shown in equations 5-9, where $x$ is defined to be the two states and $y$ is defined to be the system output while matrices A, $\mathrm{B}, \mathrm{C}, \mathrm{D}, \mathrm{G}$ and $\mathrm{H}$ represent the dynamics of the system. Furthermore, $w$ is considered as the process noise associated with the system, where $w$ is represented as $\dot{T}_{L}$, while $v$ is representing the noise associated with measurements. $L$ is represents the observer gains which are designed using the pole placement technique and $\beta$ is a parameter which is added to state space to maintain the full rank of the A matrix such that the system is observable. $\quad\left(I_{m}-\right.$ motor rotor inertia, $I_{m}-$ gear wheel inertia, $n-$ gear ratio, $k_{t}-$ motor torque constant)

$$
\begin{aligned}
& \dot{x}=\left[\begin{array}{cc}
\frac{-c_{m}}{I_{m}+I_{g} n^{2}} & \frac{-n}{I_{m}+I_{g} n^{2}} \\
0 & \beta
\end{array}\right]\left[\begin{array}{c}
\dot{\theta}_{m} \\
T_{A}
\end{array}\right]+\left[\begin{array}{c}
\frac{k_{t}}{I_{m}+I_{g} n^{2}} \\
0
\end{array}\right]\left[i_{a}\right]+\left[\begin{array}{l}
0 \\
1
\end{array}\right]\left[\dot{T}_{L}\right] \\
& \dot{\hat{x}}=A \hat{\mathrm{x}}+B u+G w+L\left(y_{t}-\hat{y}_{t}\right) \\
& \hat{y}_{t}=C \hat{\mathrm{x}} \\
& C=\left[\begin{array}{ll}
1 & 0
\end{array}\right] \\
& L=\left[\begin{array}{l}
207.34 \\
-8.92
\end{array}\right]
\end{aligned}
$$




\section{$4 \quad$ Performance Analysis}

\subsection{Estimation Assessment}

An analysis is performed to compare the actual output torque and the estimated output toque of the actuator to assess the accuracy of the estimations using full bogie vehicles when simulated using both deterministic, random tracks and measured tracks. However, only the behaviour of the first wheelset of the vehicle model is shown since other wheelsets show very similar behaviour.

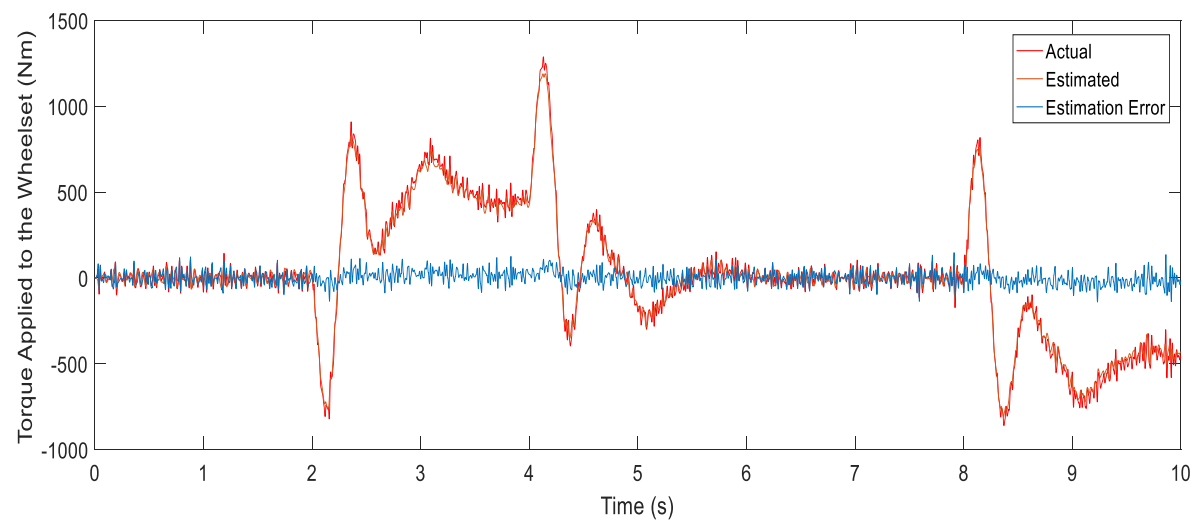

Fig. 4. Estimation Analysis for Deterministic Track - Full Bogie Vehicle

Fig. 4 shows that there are minor errors between the actual and estimated torques when full bogie vehicle is assessed. This can be clearly seen during the areas where the torque is zero and slight difference can be expected due to the process noise and noise associated with the measurement signals.

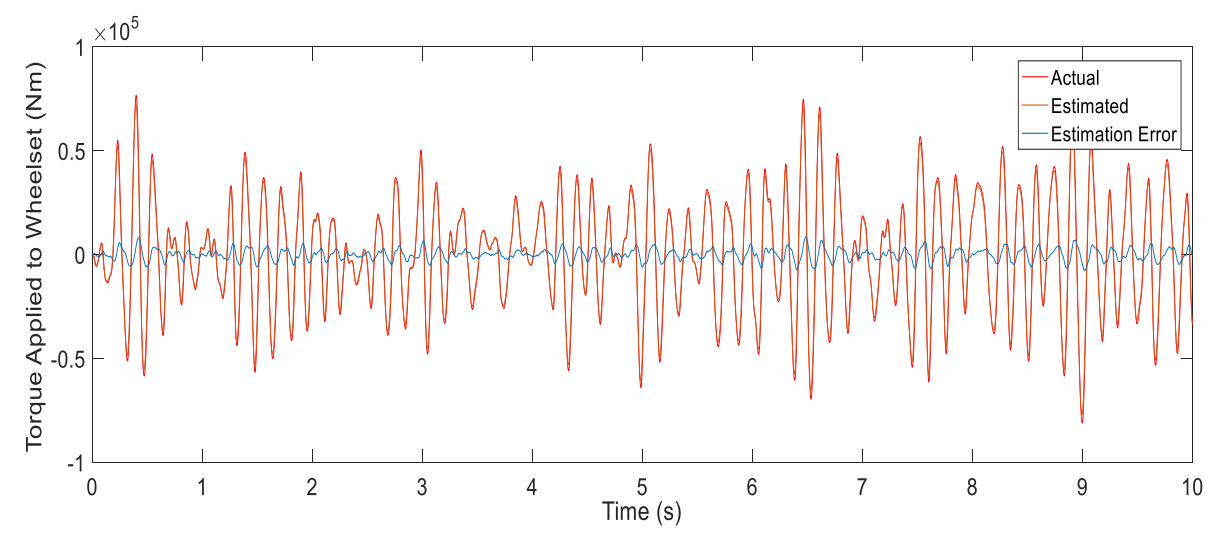

Fig. 5. Estimation Analysis for Generic Random Track - Full Bogie Vehicle 
Similar analysis is done with the random track to evaluate the observer performances and there is an approximately $10 \%$ of error between the actual and estimated torques, as it can be seen in Fig 5 .

\subsection{Robustness Assessment}

In order to assess the robustness of the observer, key parameters which are difficult to measure and define in a practical environment are varied while observing the performance of the observer. Thus, internal damping of the motor $\left(c_{m}\right)$, motor constant $\left(k_{t}\right)$ and the various inertias, such as gear wheel inertia $\left(I_{g}\right)$ and motor rotor inertia $\left(I_{m}\right)$, is being varied within a reasonable margin $( \pm 20 \%)$ to evaluate the observer performances. For this analysis, generic random track is being used while the travel speed is $83 \mathrm{~ms}^{-1}$. It is evident from Fig. 6 that the internal damping of the motor does not affect the performance of the observer within a reasonable margin.

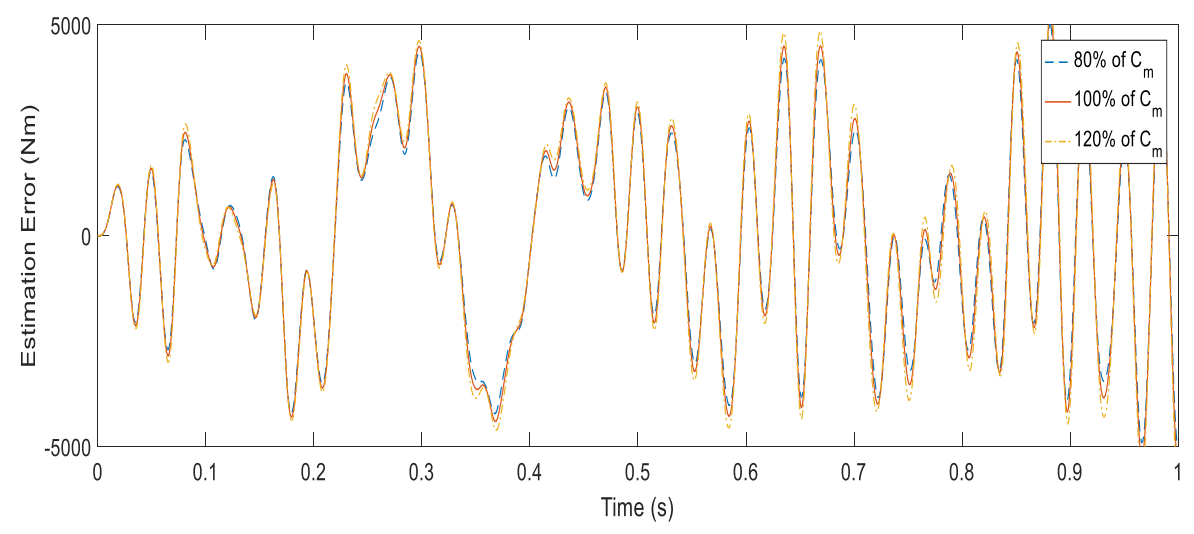

Fig. 6. Estimation Error vs. Robustness Assessment - Motor Damping

Similarly, the gear wheel inertia is varied by $\pm 20 \%$ of the value, for which it is found that observer performance is not affected by this variation as it can be seen in Fig. 7. However, as it can be seen from Fig 8, the motor rotor inertia variation does have an effect though it does not result in any detrimental performance within the operational range. 


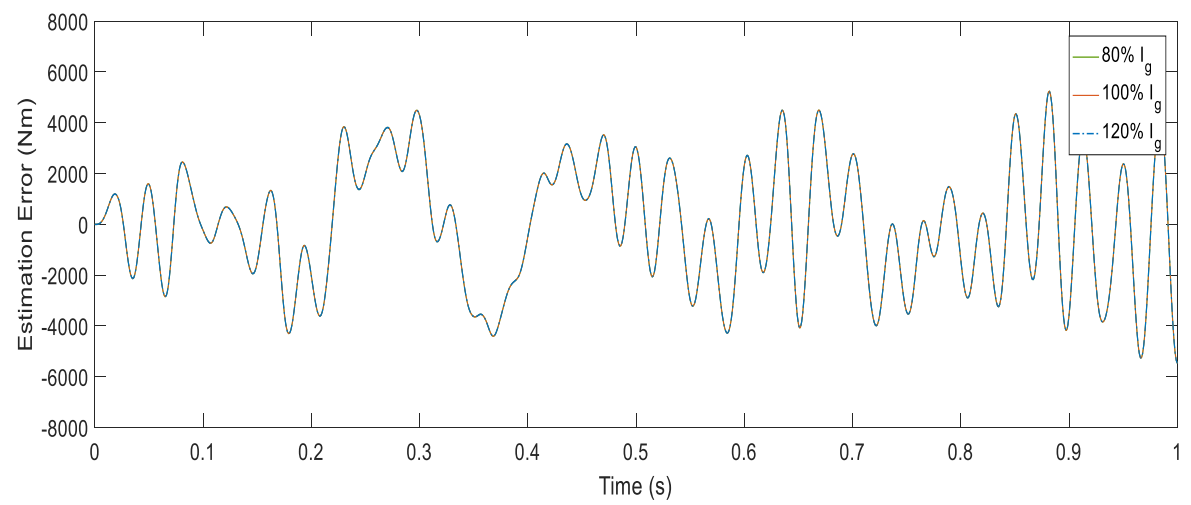

Fig. 7. Estimation Error vs. Robustness Assessment - Gear Wheel Inertia

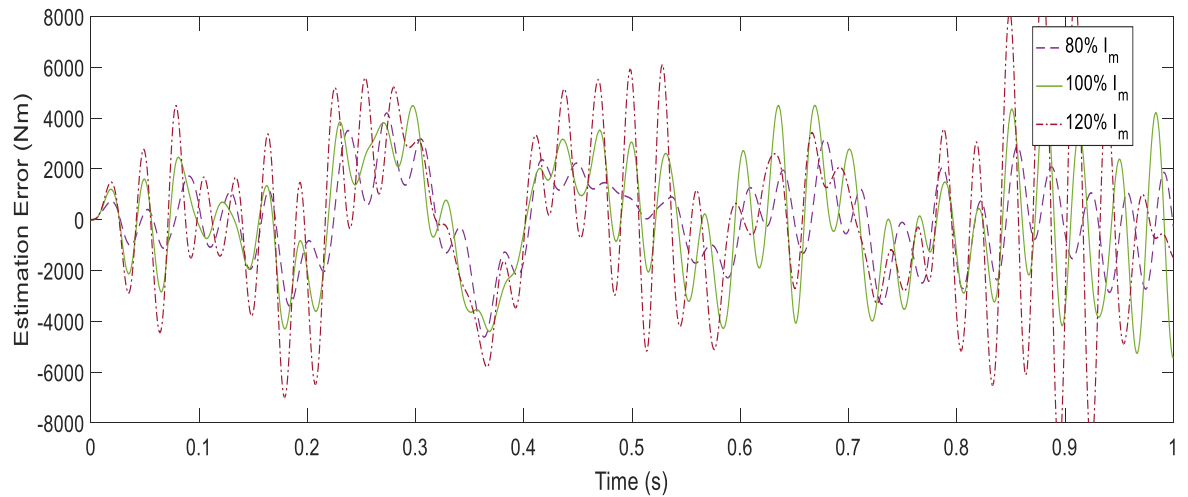

Fig. 8. Estimation Error vs. Robustness Assessment - Motor Rotor Inertia

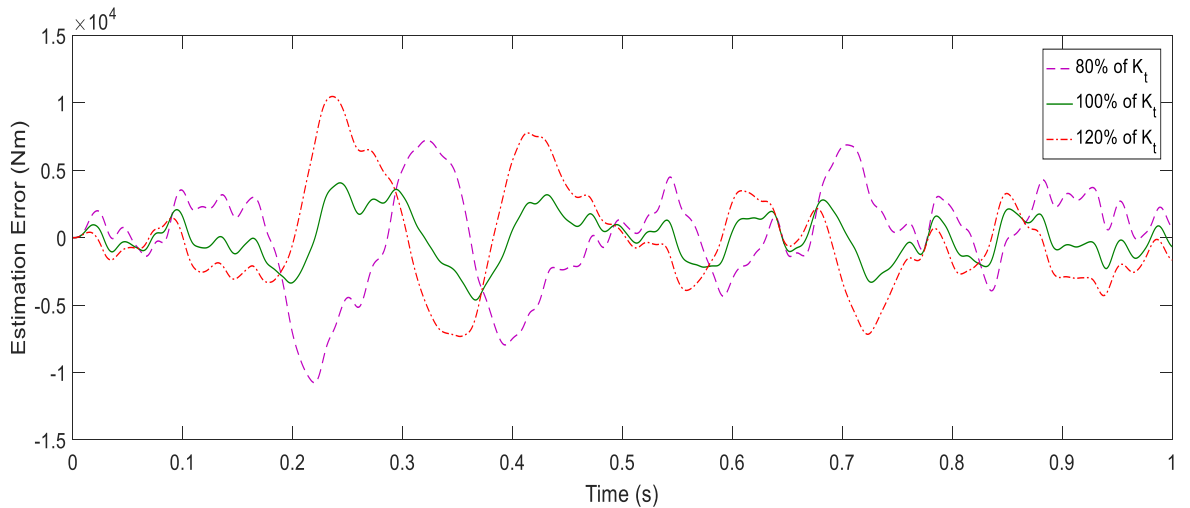

Fig. 9. Estimation Error vs. Robustness Assessment - Motor Constant - 1 
It is evident from the Fig. 9 that changing of motor constant does pose some affect to the observer performance as the error is increasing significantly (error $>100 \%$ ) as the effective value is $20 \%$ less than the original value. However, when the effective value is $20 \%$ higher, estimation error appears to rise approximately $70 \%$. Thus, observer gains require to be tuned accordingly on the cases where observer performances are compromised due to motor constant variation.

Further analysis is done to assess the observer robustness while reducing the defined value of motor constant by $10 \%$ and it can be seen from Fig. 10 that the observer maintains its robustness. It is evident form the results that the there is only a minor error increase as the effective value is being reduced by $10 \%$. Thus, it is evident that observer is robust motor constant values where it deviates $-10 \%$ to $+20 \%$ from original/defined value.

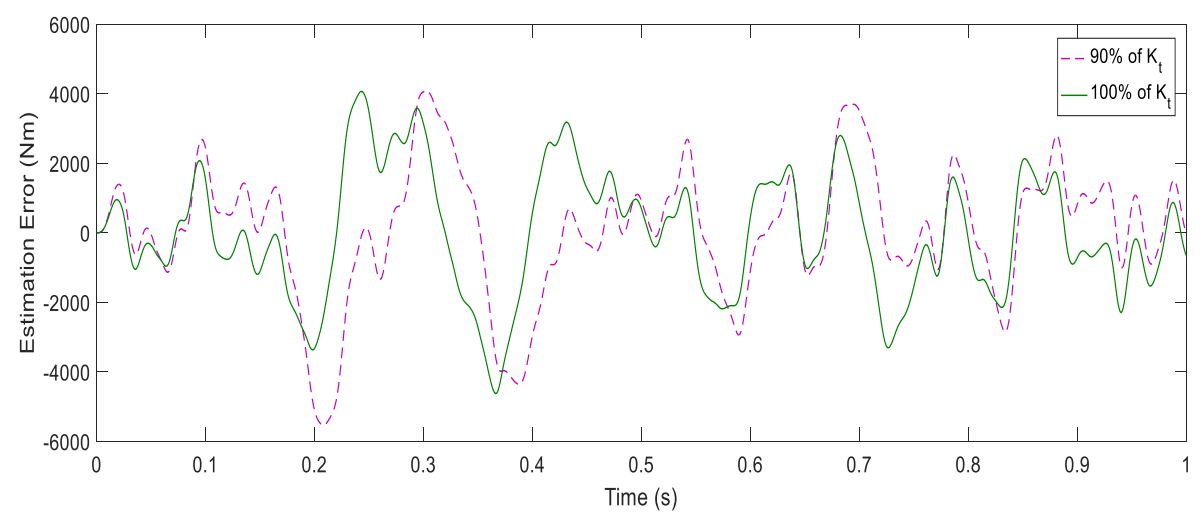

Fig. 10. Estimation Error vs. Robustness Assessment - Motor Constant - 2

These robustness assessments provides valuable insight in to the state observer performances, since, in a realistic environment, it is highly plausible that actuator parameters to be slightly different from the values used to design the sate observer. This could lead to inaccurate estimations and subsequent instability of the system. However, results of the tests are conducted indicate that the observer is capable of producing accurate estimations within a reasonable range of parameter variations since parameter variation can be expected to some extent in a realistic situation.

\section{Discussions}

As it is evident from all the results presented above, it can be concluded that the state observers can serve a significant role in this application since its capability to generate accurate estimations enables the overall system to be more practical and realistic. Analysis of the performance of the model also indicates that there is a high potential of using state estimators in active control of wheelsets with full bogie vehicle while it performed 
well in both deterministic track and measured and generic (computer generated) and measured random tracks as well. Further reviewing elaborates that estimator has adequate frequency response for a similar type other applications which deal with DC motors.

Finally, the robustness analysis re-assures the functional capabilities of the estimator under different parameter conditions and provides insight into the effect of each individual parameter.

\section{References}

1. Mei, T.X. and Goodall, R.M., "Recent Development in Active Steering of Railway Vehicles", Vehicle System Dynamics Vol 39/6, (2003).

2. T.X. Mei, H. Li and R.M. Goodall, Kalman filters applied to actively controlled railway vehicle suspensions, Transactions of the Institute of Measurement and Control 23,3 pp. 163181, (2001).

3. T.X. Mei, R.M. Goodall, H. Li, Kalman Filter for The State Estimation of A 2-Axle Railway Vehicle, European Control Conference (ECC) Germany, (1999)

4. C.P. Ward, R.M. Goodall, R. Dixon and G.A. Charles, Adhesion estimation at the wheelrail interface using advanced model based filtering, (2012).

5. Hongbin Ren, Sizhong Chen, Yuzhuang Zhao, Gang Liu \& Lin Yang State observer-based sliding mode control for semi-active hydro-pneumatic suspension, Vehicle System Dynamics, 54:2, 168-190, (2016).

6. Stefano Bruni, Roger Goodall, T. X. Mei and Hitoshi Tsunashima, Control and monitoring for railway vehicle dynamics - Vehicle System Dynamics International Journal of Vehicle Mechanics and Mobility, (2007).

7. Pearson, J.T., Goodall, R.M., Mei, T.X. and Himmelstein, G, Active stability control strategies for a high speed bogie. Control Engineering Practice, 12, 1381-1391 (2004).

8. T.X. Mei And R.M. Goodall, Modal Controllers for Active Steering of Railway Vehicles with Solid Axle Wheelsets, (2000).

9. T. X. Mei and R. M. Goodall, Stability control of railway bogies using absolute stiffness skyhook spring approach, (2006).

10. Shen, G. and Goodall, R.M., Active yaw relaxation for improved bogie performance. Vehicle System Dynamics, 28, 273 - 289, (1997) 